\title{
The Existence and Uniqueness of Global Solutions to the Initial Value Problem for the System of Nonlinear Integropartial Differential Equations in Spatial Economics: The Dynamic Continuous Dixit-Stiglitz-Krugman Model in an Urban-Rural Setting
}

\author{
Minoru Tabata ${ }^{1}$ and Nobuoki Eshima ${ }^{2}$ \\ ${ }^{1}$ Department of Mathematical Sciences, Osaka Prefecture University, Sakai, Osaka 599-8531, Japan \\ ${ }^{2}$ Department of Statistics, Oita University, Oita 879-5593, Japan \\ Correspondence should be addressed to Minoru Tabata; mnrtabata@luck.ocn.ne.jp
}

Received 18 May 2014; Accepted 29 July 2014

Academic Editor: Juan J. Nieto

Copyright ( $) 2015$ M. Tabata and N. Eshima. This is an open access article distributed under the Creative Commons Attribution License, which permits unrestricted use, distribution, and reproduction in any medium, provided the original work is properly cited.

\begin{abstract}
Assume that economic activities are conducted in a bounded continuous domain where workers move toward regions that offer higher real wages and away from regions that offer below-average real wages. The density of real wages is calculated by solving the nominal wage equation of the continuous Dixit-Stiglitz-Krugman model in an urban-rural setting. The evolution of the density of workers is described by an unknown function of the replicator equation whose growth rate is equal to the difference between the density of real wages and the average real wage. Hence, the evolution of the densities of workers and real wages is described by the system of the nominal wage equation and the replicator equation. This system of equations is an essentially new kind of system of nonlinear integropartial differential equations in the theory of functional equations. The purpose of this paper is to obtain a sufficient condition for the initial value problem for this system to have a unique global solution.
\end{abstract}

\section{Introduction}

The new economic geography (NEG) is a new branch of spatial economics that was initiated by Krugman in the early 1990s. This new branch has attracted many social scientists and becomes one of the most important major branches of spatial economics at present. In 2008 Krugman received the Nobel Memorial Prize in Economic Sciences (officially Sveriges Riksbank Prize in Economic Sciences in Memory of Alfred Nobel) for his great contribution to the NEG (see [1-6]). A large number of mathematical models have been built in the NEG. In particular there are many models described by nonlinear integropartial differential equations that are new and important in the theory of functional equations (see [1$3]$ ). Hence the NEG is regarded as a new frontier of the theory of nonlinear integropartial differential equations.

The Krugman core-periphery model (the CP model) is the origin of the NEG (see [1, Chapter 5]) since various models are constructed as its extension. The $\mathrm{CP}$ model is a discrete model. In this model economic activities are conducted at two points. These two points represent a core region and a periphery region, respectively. An extension to the case of a finite set of points has been studied in [1]. This model is called the Dixit-Stiglitz-Krugman model (DSK model). Its mathematical foundation is studied in [7-13]. Moreover, in [14], we consider an extension of the CP model to the case of a bounded continuous domain where economic activities are conducted continuously in space. This model is called the continuous DSK model (cDSK model).

These models are static models with no population dynamics. It is very important in spatial economics to build population dynamics into them. Hence Krugman constructs the dynamic DSK model (dDSK model) by combining the DSK model with the replicator dynamics that is one of the most fundamental dynamics in evolutionary game theory 
(see [15] and [16, Chapter 3]). His dynamic model is very important in spatial economics since it describes economies of agglomeration in the case where workers move from one point to another to seek higher real wages within a finite set of points at which economic activities are conducted (see [1, p. 62, p. 77]). Hence, by following this line, we consider the dynamic cDSK model (dcDSK model) in this paper; that is, we combine the cDSK model with the replicator dynamics. This dynamic model is regarded as a continuous version of the dDSK model and explains agglomeration of capital and concentration of workers when workers move in a bounded continuous domain where economic activities are conducted continuously in space.

Let us discuss the dcDSK model from the viewpoint of the theory of functional equations. The dcDSK model is described by the system of the nominal wage equation and the replicator equation. We refer to this system of equations as the dcDSK system. The nominal wage equation is a nonlinear integral equation that contains the density of nominal wages as an unknown function and the density of workers as a known function (see [14, (2.4)]). Hence, if we solve this equation under the condition that the density of workers is a given function, then we can obtain the density of nominal wages. However, the integral kernel of the nominal wage equation contains not only these densities but also the price index. We must note that the price index itself is a nonlinear integral operator acting on the density of nominal wages and the density of workers (see [14, (2.7)]). Therefore, we can say that the nominal wage equation is a double nonlinear integral equation (see [14, Remark 2.3] for mathematical difficulties caused by the double nonlinearity).

The replicator equation is a nonlinear integropartial differential equation whose unknown function denotes the density of workers. Its coefficient denotes the growth rate of worker population (see $[1,(5.1),(5.2)]$ and $[16$, p. 73]). The coefficient is equal to the difference between the density of real wages and the average real wage, where the density of real wages is defined as the density of nominal wages deflated by a fractional power of the price index, and the average real wage is defined as the integral of the product of the density of workers and the density of real wages (see [1, $(5,1),(5.6)])$. Hence, the coefficient is regarded as a double nonlinear integral operator acting on the density of workers and the density of nominal wages.

Moreover, the coefficient of the replicator equation of the dcDSK system contains an unknown function implicitly in the sense that the coefficient is determined by solving the nominal wage equation under the condition that the unknown function is given, in contrast to the replicator equation whose coefficient explicitly contains an unknown function in evolutionary game theory (see $[16,(3.3)])$. If we can define an operator that maps the density of workers to the density of real wages by solving the nominal wage equation under the condition that the density of workers is a given function, then the replicator equation is regarded as a nonlinear integropartial differential equation whose coefficient contains the operator that acts on an unknown function.
For these reasons we deduce that the dcDSK system is an essentially new kind of system of nonlinear integropartial differential equations. Therefore, it is important to study this system not only in spatial economics but also in the theory of functional equations. In this paper we prove a sufficient condition for the initial value problem for the dcDSK system to have a unique global solution and obtain estimates of the solution. The main result is Theorem 4 .

\section{The System of Equations}

Let us introduce the notations. Let $E$ be a domain of a Euclidean space. By $L^{1}(E)$ we denote the Banach space of all Lebesgue summable functions of $x \in E$. By $L^{\infty}(E)$ we denote the Banach space of all essentially bounded functions of $x \in E$. By $C(E)$ we denote the Banach space of all uniformly bounded continuous functions of $x \in E$.

We assume that economic activities are conducted continuously in a bounded domain $D$ of an $m$-dimensional Euclidean space, where $m$ is a positive integer. If $m \geq 3$, then the model is unrealistic from the viewpoint of economics, but we accept such a case for mathematical generality in this paper. We denote the norms of the Banach spaces $L^{1}(D)$ and $C(D)$ by $\|\cdot \cdot\| \mid$ and $\|\cdot\|$, respectively; that is, we define

$$
\||u(\cdot)|\|:=\int_{y \in D}|u(y)| d y, \quad\|u(\cdot)\|:=\sup _{y \in D}|u(y)| .
$$

Let $T \geq 0$. By $L^{\infty, 1}([0, T] \times D)$ we denote the Banach space of all functions $h=h(t, x)$ such that

$$
\underset{0 \leq t \leq T}{\operatorname{ess} \sup }\|h(t, \cdot)\|<+\infty \text {. }
$$

By $L^{1}{ }_{+}(E), L^{\infty}{ }_{+}(E), C_{+}(E)$, and $L^{\infty, 1}{ }_{+}([0, T] \times D)$ we denote the set of all positive-valued functions of $L^{1}(E), L^{\infty}(E), C(E)$, and $L^{\infty, 1}([0, T] \times D)$, respectively. By $L^{1}{ }_{0+}(E), L^{\infty}{ }_{0+}(E)$, $C_{0+}(E)$, and $L^{\infty, 1}{ }_{0+}([0, T] \times D)$ we denote the set of all nonnegative-valued functions of $L^{1}(E), L^{\infty}(E), C(E)$, and $L^{\infty, 1}([0, T] \times D)$, respectively.

Let us introduce the dcDSK system. The nominal wage equation is a nonlinear integral equation of the following form (see $[1,(5.5)]$ and $[14,(2.4)])$ :

$$
\begin{aligned}
& w(t, x)^{\sigma} \\
& =\int_{y \in D} Y_{\mu}(\lambda(t, y), w(t, y)) G_{\sigma}(\lambda(t, \cdot), w(t, \cdot) ; y)^{\sigma-1} \\
& \cdot e^{-(\sigma-1) c(x, y)} d y,
\end{aligned}
$$

where $w=w(t, x)$ is an unknown function that denotes the density of nominal wages at time $t \geq 0$ and at point $x \in D$. By $G_{\sigma}=G_{\sigma}(\lambda(t, \cdot), w(t, \cdot) ; x)$ we denote the price index, which is a nonlinear integral operator of the following form (see $[1$, (5.4)] and $[14,(2.7)])$ :

$$
\begin{aligned}
G_{\sigma} & (\lambda(t, \cdot), w(t, \cdot) ; x)^{\sigma-1} \\
& :=\frac{1}{\int_{y \in D} \lambda(t, y)(1 / w(t, y))^{\sigma-1} e^{-(\sigma-1) c(x, y)} d y},
\end{aligned}
$$


where $\lambda=\lambda(t, y)$ represents the density of workers at time $t \geq 0$ and at point $y \in D$. By $\sigma$ we denote the elasticity of substitution among varieties of manufactured goods. We assume that

$$
\sigma>1
$$

When $\sigma$ increases, varieties of manufactured goods are close to perfect substitutes; as $\sigma$ decreases toward 1 , the desire to consume a greater variety of manufactured goods increases (see [1, p. 46] and [2, p. 308]). We denote the income at time $t \geq 0$ and at point $y \in D$ by $Y_{\mu}=Y_{\mu}(\lambda(t, y), w(t, y))$. The dcDSK model consists of a monopolistically competitive sector (manufacturing) and a perfectly competitive sector (agriculture) (see [1, p. 61]). Hence the income consists of agricultural income and manufacturing income; that is, it has the following form (see $[1,(5.3)])$ :

$$
\begin{gathered}
Y_{\mu}(\lambda(t, y), w(t, y))=\mu \lambda(t, y) w(t, y)+(1-\mu) \phi(y) \\
0<\mu<1
\end{gathered}
$$

where $\mu$ and $(1-\mu)$ denote the share of manufacturing expenditure and the share of agricultural expenditure, respectively, and we denote the density of farmers at point $y \in D$ by $\phi=\phi(y)$. We assume that $\phi=\phi(y)$ is a given function such that (see $[14,(2.12),(2.14)])$

$$
\begin{gathered}
\phi(y) \in L_{0+}^{1}(D), \\
\|\phi(\cdot) \mid\|=1 .
\end{gathered}
$$

Note that this function is independent of the time variable $t \geq 0$.

The function $c=c(x, y)$ represents the iceberg form of transport costs (see [17]). We refer to this function as the transport cost function. We reasonably accept the following assumption (see [14, Assumption 2.1]).

Assumption 1. The transport cost function $c=c(x, y)$ is a nonnegative-valued continuous function of $(x, y) \in D \times D$ such that

$$
\begin{gathered}
c(x, x)=0 \quad \text { for each } x \in D, \\
c(x, y)=c(y, x) \quad \text { for each } x, y \in D, \\
c(x, y)>0 \quad \text { if } x \neq y, \\
\mathbf{C}:=\sup _{(x, y) \in D \times D} c(x, y)<+\infty .
\end{gathered}
$$

Considering (6), and noting that the right-hand side of (4) is a nonlinear integral operator acting on the density of workers $\lambda=\lambda(t, x)$ and the density of nominal wages $w=w(t, x)$, we see that the right-hand side of (3) is a double nonlinear integral operator acting on these densities.

We define the density of real wages $\omega=\omega(t, x)$ at time $t \geq 0$ and at point $x \in D$ by deflating the density of nominal wages by a fractional power of the price index as follows (see $[1,(5.6)],(4)$, and (7)):

$$
\omega=\omega(t, x):=\frac{w(t, x)}{G_{\sigma}(\lambda(t, \cdot), w(t, \cdot) ; x)^{\mu}} .
$$

The density of real wages can be regarded as a nonlinear integral operator acting on $\lambda=\lambda(t, x)$ and $w=w(t, x)$.

The replicator equation is a nonlinear integropartial differential equation of the following form (see $[1,(5.2)]$ and $[13,(2.22)])$ :

$$
\left(\frac{\partial}{\partial t}\right) \lambda(t, x)=a M(\lambda(t, \cdot), \omega(t, \cdot) ; x) \lambda(t, x),
$$

where $a$ denotes a positive constant. We define

$$
M(\lambda(t, \cdot), \omega(t, \cdot) ; x):=\omega(t, x)-m(\lambda(t, \cdot), \omega(t, \cdot)),
$$

where $m(\lambda(t, \cdot), \omega(t, \cdot))$ is the average real wage defined as follows in the same way as $[1,(5.1)]$ and $[13,(2.23)]$ :

$$
m(\lambda(t, \cdot), \omega(t, \cdot)):=\int_{y \in D} \lambda(t, y) \omega(t, y) d y .
$$

It follows from (11), (12), and (13) that workers move toward regions that offer higher real wages and away from regions that offer below-average real wages (see [1, p. 62]). Considering (4) and (10), we see that (12) is a double nonlinear integral operator acting on $\lambda=\lambda(t, x)$ and $w=w(t, x)$. In this paper for simplicity we assume that

$$
a=1 \text {. }
$$

Hence (12) is the growth rate. The dcDSK system consists of (3), (10), and (11). In Section 4 we define an operator that maps $\lambda=\lambda(t, x)$ to $\omega=\omega(t, x)$ by solving (3) under the condition that $\lambda=\lambda(t, x)$ is a given function. Substituting this operator in (11), we can transform the dcDSK system into the replicator equation whose coefficient contains the operator that maps $\lambda=\lambda(t, x)$ to $\omega=\omega(t, x)$.

\section{Result and Discussion}

We consider the initial value problem by imposing the following initial condition on the dcDSK system (3), (10), and (11):

$$
\lambda(0, x)=\lambda^{0}(x) \text { for a.e. } x \in D \text {, }
$$

where $\lambda^{0}=\lambda^{0}(x)$ is a given function of $x \in D$. This function denotes the initial density of workers. The following assumption is imposed on this function in [1, pp. 61-63] and $[14,(2.11),(2.13)]$.

Assumption 2. (i) $\lambda^{0}=\lambda^{0}(x) \in L^{1}{ }_{0+}(D)$.

(ii) $\left\||| \lambda^{0}(\cdot) \mid\right\|=1$.

Hence, we accept this assumption in this paper also. Let $T>0$ be a constant. If a function

$$
(\lambda, \omega, w)=(\lambda(t, x), \omega(t, x), w(t, x))
$$

belongs to

$$
L_{0+}^{\infty, 1}([0, T] \times D) \times L_{+}^{\infty}([0, T] \times D) \times L_{+}^{\infty}([0, T] \times D)
$$


and satisfies the dcDSK system for a.e. $(t, x) \in[0, T] \times D$ and the initial condition (15), then we say that the function (16) is a solution to the initial value problem in $[0, T]$. If a function (16) is defined for a.e. $(t, x) \in[0,+\infty) \times D$ and is a solution to the initial value problem in $[0, T]$ for each $T>0$, then we say that the solution is global. No boundary condition needs to be imposed on the density of workers, since the evolution of the density of workers can be determined uniquely in (17) by the initial condition (15) as done in Section 5.

We define a function $V=V(\mu, \sigma),(\mu, \sigma) \in(0,1) \times$ $(1,+\infty)$ in order to state a sufficient condition for the initial value problem to have a unique global solution. Consider the quadratic equation

$$
I(\mu, \sigma ; u):=\left(1-\frac{1}{\sigma}\right) u^{2}+\left(\frac{\mu}{\sigma}\right) u-1=0,
$$

where $u$ denotes an unknown quantity. It follows from (5) that this equation has a positive solution and a negative solution. We denote the positive solution by $U=U(\mu, \sigma)$. We see easily that

$$
U(\mu, \sigma)=\frac{\left\{-\mu+\left(\mu^{2}+4 \sigma(\sigma-1)\right)^{1 / 2}\right\}}{(2(\sigma-1))}
$$

By making use of this positive solution, we define the following quadratic equation:

$$
J(\mu, \sigma ; v):=v^{2}+\mu\left(U(\mu, \sigma)^{1 / \sigma}-1\right) v-U(\mu, \sigma)^{1 / \sigma}=0,
$$

where $v$ denotes an unknown quantity. This quadratic equation has a positive solution and a negative solution, since $U(\mu, \sigma)^{1 / \sigma}>0$. We denote the positive solution by $V=$ $V(\mu, \sigma)$. We see easily that

$$
\begin{aligned}
V=V & (\mu, \sigma) \\
:= & \left\{-\mu\left(U(\mu, \sigma)^{1 / \sigma}-1\right)\right. \\
& \left.+\left(\mu^{2}\left(U(\mu, \sigma)^{1 / \sigma}-1\right)^{2}+4 U(\mu, \sigma)^{1 / \sigma}\right)^{1 / 2}\right\} \cdot 2^{-1} .
\end{aligned}
$$

The following lemma is proved in [12, Lemma 3.2] (see [12, (3.3)-(3.8)]).

Lemma 3. (i) $1<V(\mu, \sigma)<1 / \mu$ for each $(\mu, \sigma) \in(0,1) \times$ $(1,+\infty)$.

(ii) $V=V(\mu, \sigma)$ is a strictly monotone-decreasing smooth function of $\sigma>1$ for each $\mu \in(0,1)$.

(iii) $V=V(\mu, \sigma)$ is a strictly monotone-decreasing smooth function of $\mu \in(0,1)$ for each $\sigma>1$.

(iv) $\lim _{\sigma \rightarrow 1+0} V(\mu, \sigma)>1, \lim _{\sigma \rightarrow+\infty} V(\mu, \sigma)=1$, for each $\mu \in(0,1)$.

(v) $\lim _{\mu \rightarrow 0+0} V(\mu, \sigma)>1, \lim _{\mu \rightarrow 1-0} V(\mu, \sigma)=1$, for each $\sigma>1$
The following theorem is the main result of this paper, which is proved in Sections 4 and 5 (see Assumption 1).

Theorem 4. If $\mu, \sigma$, and $\mathrm{C}$ satisfy (5), (7), and the following inequality:

$$
\mathbf{C}<\frac{\{\log (V(\mu, \sigma))\}}{(\sigma-1)},
$$

then the initial value problem has a unique global solution $(\lambda, \omega, w)=(\lambda(t, x), \omega(t, x), w(t, x))$, and this solution satisfies the following:

$$
|||\lambda(r, \cdot)-\lambda(s, \cdot)||| \leq \mathbf{a}|r-s| \quad \text { for each } r, s \geq 0,
$$

$\lambda=\lambda(t, x)$ is continuous at $t=0+0$

and partially differentiable for $t>0$ for a.e. $x \in D$,

$$
\omega=\omega(t, x), \quad w=w(t, x) \in C_{+}(D) \quad \text { for each } t \geq 0
$$

$$
\begin{array}{r}
\|w(r, \cdot)-w(s, \cdot)\| \leq \delta_{1}(\mu, \sigma, \mathbf{C})|r-s|, \\
\|\omega(r, \cdot)-\omega(s, \cdot)\| \leq \delta_{2}(\mu, \sigma, \mathbf{C})|r-s|
\end{array}
$$

for each $r, s \geq 0$,

$$
\begin{gathered}
\lambda^{0}(x) \exp (-\mathbf{a} t) \leq \lambda(t, x) \leq \lambda^{0}(x) \exp (\mathbf{a} t) \\
\text { for each } t \geq 0 \text { and a.e. } x \in D, \\
\|\| \lambda(t, \cdot) \|=1 \quad \text { for each } t \geq 0,
\end{gathered}
$$

$\frac{1}{\alpha_{+}} \leq w(t, x) \leq \frac{1}{\alpha_{-}} \quad$ for each $(t, x) \in[0,+\infty) \times D$,

$$
\begin{gathered}
\frac{1}{\alpha_{+}} \leq G_{\sigma}(\lambda(t, \cdot), w(t, \cdot) ; x) \leq\left(\frac{1}{\alpha_{-}}\right) \exp (\mathbf{C}) \\
\text { for each }(t, x) \in[0,+\infty) \times D, \\
\mathbf{a}_{1} \leq \omega(t, x) \leq \mathbf{a}_{2} \quad \text { for each }(t, x) \in[0,+\infty) \times D, \\
-\mathbf{a} \leq M(\lambda(t, \cdot), \omega(t, \cdot) ; x) \leq \mathbf{a} \\
\text { for each }(t, x) \in[0,+\infty) \times D,
\end{gathered}
$$

where $\delta_{i}=\delta_{i}(\mu, \sigma, \mathbf{C}), i=1,2$, are positive-valued functions of $(\mu, \sigma, \mathbf{C}) \in(0,1) \times(1,+\infty) \times(0,+\infty)$ and

$$
\begin{gathered}
\mathbf{a}_{1}:=\frac{(\exp (-\mu \mathrm{C})) \alpha_{-}{ }^{\mu}}{\alpha_{+}}, \\
\mathbf{a}_{2}:=\frac{\alpha_{+}{ }^{\mu}}{\alpha_{-}}, \quad \mathbf{a}:=\mathbf{a}_{2}-\mathbf{a}_{1}, \\
\alpha_{ \pm}:=\frac{\{\exp ( \pm(\sigma-1) \mathbf{C})-\mu\}}{(1-\mu)} .
\end{gathered}
$$

Let us discuss this theorem. The inequalities (5), (7), and (22) are a sufficient condition for the initial value problem 
to have a unique global solution. Note that $V=V(\mu, \sigma)$ is independent of C. Making use of Lemma 3, (i), (ii), (iv), we deduce that

if $\sigma>1$ and $\mathbf{C}>0$ are sufficiently small, then (22) holds.

Hence we can say that (22) holds for a much larger set of $(\mu, \sigma, \mathbf{C}) \in(0,1) \times(1,+\infty) \times(0,+\infty)$. By Lemma 3, (i), we see that (5), (7), and (22) imply the following inequality:

$$
\mathbf{C}<\frac{(\log (1 / \mu))}{(\sigma-1)} .
$$

Applying this inequality, Assumption 1, (5), and (7) to (33) and (34), we see that

$$
\begin{aligned}
& 0<\alpha_{-}<1<\alpha_{+}, \\
& 0<\mathbf{a}_{1}<1<\mathbf{a}_{2} .
\end{aligned}
$$

Making use of (5), (7), (8), and the above theorem, we see easily that the right-hand sides of (3), (4), and (12) belong to $L^{\infty}([0, T] \times D)$ for each $T>0$ and that the right-hand side of (11) belongs to $L^{\infty, 1}([0, T] \times D)$ for each $T>0$. The conservation law of workers follows from (17) and (28). It follows from (27) that

$$
\begin{array}{r}
\{x \in D ; \lambda(t, x)=0\}=\left\{x \in D ; \lambda^{0}(x)=0\right\} \\
\text { for each } t \geq 0 .
\end{array}
$$

Hence, no worker moves toward a point where no worker lives. Combining (25) and (26), we see that

$$
\begin{array}{r}
\omega=\omega(t, x), \quad w=w(t, x) \in C_{+}([0, T] \times D) \\
\text { for each } T>0 .
\end{array}
$$

The functions $\delta_{i}=\delta_{i}(\mu, \sigma, \mathbf{C}), i=1,2$, are defined in Lemma 17.

Remark 5. We impose (7) on the dcDSK model; that is, we consider the dcDSK model in an urban-rural setting. In this paper we cannot treat the case

$$
\mu=1
$$

that is, we cannot consider the dcDSK model in an urban setting (see [1, p. 331]) because it follows from Lemma 3, (i), that (41) cannot be substituted in (22). The DSK model with (41) is studied in [13]. The cDSK model with (41) is studied in [14, Theorem 3.2].

\section{Solutions of the Nominal Wage Equation}

Let us solve the nominal wage equation (3) under the condition that the density of workers is a given function. In this section we do not deal with the replicator equation. Hence, we have no need to consider the time evolution of the density of workers, the density of nominal wages, and the density of real wages. Therefore, for simplicity of symbols, we omit the time variable $t$ from these densities, and we denote them by $\lambda=\lambda(x), w=w(x)$, and $\omega=\omega(x)$ in (3), (4), (6), and (10) in this section. We refer to these equations with the same numbers. No confusion should arise. We assume that $\lambda=\lambda(x)$ is a given function that satisfies the same condition as Assumption 2 as follows:

$$
\begin{gathered}
\lambda(x) \in L_{0+}^{1}(D), \\
\|\| \lambda(\cdot)\|\|=1 .
\end{gathered}
$$

Proposition 6. If $\mu, \sigma$, and $\mathbf{C}$ satisfy (5), (7), and (36), then the following statements ( $i$ ) and (ii) hold.

(i) The nominal wage equation (3) has a solution $w=$ $w(x)$ in $L_{+}^{\infty}(D)$.

(ii) If (3) has a solution $w=w(x)$ in $L^{\infty}{ }_{+}(D)$, then the solution satisfies the following:

$$
\begin{gathered}
w=w(x), \quad \omega=\omega(x) \in C_{+}(D), \\
\frac{1}{\alpha_{+}} \leq w(x) \leq \frac{1}{\alpha_{-}} \quad \text { for each } x \in D, \\
\frac{1}{\alpha_{+}} \leq G_{\sigma}(\lambda(\cdot), w(\cdot) ; x) \leq\left(\frac{1}{\alpha_{-}}\right) \exp (\mathbf{C}) \\
\quad \text { for each } x \in D, \\
\quad \text { for each } x \in D, \\
\mathbf{a}_{1} \leq \omega(x) \leq \mathbf{a}_{2}, \quad \mathbf{a}_{1} \leq m(\lambda(\cdot), \omega(\cdot)) \leq \mathbf{a}_{2}, \\
\text { for each } x \in D(\lambda(\cdot), \omega(\cdot) ; x) \leq \mathbf{a} .
\end{gathered}
$$

Proof. In [14, Theorem 3.1, (i), (ii)], from (5), (7), and (36), we prove (i), and we prove that if (3) has a solution in $L^{\infty}{ }_{+}(D)$, then the solution satisfies (44)-(47). Applying (42), (43), and (47) to (12) and (13), we obtain (48) and (49).

Lemma 7. Let $r$ and $s$ be positive constants. Let $\beta \in \mathbb{R}$ be a constant. If $g_{i}=g_{i}(x) \in C(D), i=1,2$, satisfy the following inequality:

$$
r \leq g_{i}(x) \leq s \quad \text { for each } x \in D, i=1,2,
$$

then

$$
\left\|g_{1}(\cdot)^{\beta}-g_{2}(\cdot)^{\beta}\right\| \leq h(\beta, r, s)\left\|g_{1}(\cdot)-g_{2}(\cdot)\right\|,
$$

where

$$
\begin{aligned}
& h(\beta, r, s):=\beta s^{\beta-1} \quad \text { if } \beta>1, \\
& h(\beta, r, s):=|\beta| r^{\beta-1} \quad \text { if } \beta \leq 1 .
\end{aligned}
$$

Proof. By the mean value theorem we see easily that if $0<$ $X_{1} \leq X_{2}$, then there exists a constant $\xi \in\left[X_{1}, X_{2}\right]$ such that

$$
X_{1}^{\beta}-X_{2}^{\beta}=\beta \xi^{\beta-1}\left(X_{1}-X_{2}\right) \text {. }
$$


Substituting $\left(X_{1}, X_{2}\right)=\left(g_{1}(y), g_{2}(y)\right)$ or $\left(g_{2}(y), g_{1}(y)\right)$ in this equality and making use of $(50)$, we see that

$$
\left|g_{1}(y)^{\beta}-g_{2}(y)^{\beta}\right| \leq|\beta| \max _{r \leq \xi \leq s} \xi^{\beta-1}\left|g_{1}(y)-g_{2}(y)\right|
$$

Applying (52) to the right-hand side of this inequality, we obtain the present lemma.

Lemma 8. Assume that $\mu, \sigma$, and $\mathbf{C}$ satisfy (5), (7), and (22). If $\lambda_{i}=\lambda_{i}(x), i=1,2$, satisfy (42) and (43), and $w_{i}=$ $w_{i}(x) \in C_{+}(D)$ is a solution of (3) with $\lambda(x)=\lambda_{i}(x), i=1,2$, respectively, then

$$
\left\|w_{1}(\cdot)-w_{2}(\cdot)\right\| \leq\left\|\left|\lambda_{1}(\cdot)-\lambda_{2}(\cdot) \|\right| \gamma_{1}(\mu, \sigma, \mathbf{C}),\right.
$$

where $\gamma_{1}=\gamma_{1}(\mu, \sigma, \mathbf{C})$ is a positive-valued function of $(\mu, \sigma, \mathbf{C}) \in(0,1) \times(1,+\infty) \times(0,+\infty)$.

Proof. Let us transform the nominal wage equation (3). Define the following new unknown function:

$$
W=W(x):=w(x)^{\sigma}
$$

Defining the following nonlinear integral operator:

$$
\begin{aligned}
& H(\lambda(\cdot), W(\cdot) ; x) \\
& \quad:=\int_{y \in D} \lambda(y)\left(\frac{1}{W(y)}\right)^{1-1 / \sigma} e^{-(\sigma-1) c(x, y)} d y,
\end{aligned}
$$

we rewrite (4) as follows:

$$
G_{\sigma}(\lambda(\cdot), w(\cdot) ; x)^{\sigma-1}=\frac{1}{H(\lambda(\cdot), W(\cdot) ; x)}
$$

Making use of this equality and defining the following nonlinear integral operator (see (6)):

$$
\begin{aligned}
& F(\lambda(\cdot), W(\cdot) ; x) \\
& :=\int_{y \in D}\left\{\frac{Y_{\mu}\left(\lambda(y), W(y)^{1 / \sigma}\right)}{H(\lambda(\cdot), W(\cdot) ; y)}\right\} e^{-(\sigma-1) c(x, y)} d y,
\end{aligned}
$$

we rewrite (3) equivalently as follows:

$$
W(x)=F(\lambda(\cdot), W(\cdot) ; x) .
$$

Hence, we see that

$$
W_{i}(x)=F\left(\lambda_{i}(\cdot), W_{i}(\cdot) ; x\right), \quad i=1,2,
$$

where $W_{i}=W_{i}(x)$ is defined by $w_{i}=w_{i}(x)$ in the same way as (56), $i=1,2$. Subtract both sides of (61) with $i=2$ from those of (61) with $i=1$. The right-hand side of the equality thus obtained is transformed as follows (see (6)):

$$
\begin{aligned}
& W_{1}(x)-W_{2}(x) \\
& =\int_{y \in D} \mu\left(\lambda_{1}(y)-\lambda_{2}(y)\right) W_{1}(y)^{1 / \sigma} \\
& +\left(\frac{1}{H\left(\lambda_{1}(\cdot), W_{1}(\cdot) ; y\right)}\right) e^{-(\sigma-1) c(x, y)} d y \\
& +\int_{y \in D} \mu \lambda_{2}(y)\left(W_{1}(y)^{1 / \sigma}-W_{2}(y)^{1 / \sigma}\right) \\
& \quad+\int_{y \in D} Y_{\mu}\left(\lambda_{2}(y), W_{2}(y)^{1 / \sigma}\right) \Delta_{1}(y) e^{-(\sigma-1) c(x, y)} d y \\
& \quad+\int_{y \in D} Y_{\mu}\left(\lambda_{2}(y), W_{2}(y)^{1 / \sigma}\right) \Delta_{2}(y) e^{-(\sigma-1) c(x, y)} d y
\end{aligned}
$$

where

$$
\begin{array}{r}
\Delta_{i}(y):=\frac{1}{H\left(\lambda_{1}(\cdot), W_{i}(\cdot) ; y\right)}-\frac{1}{H\left(\lambda_{i}(\cdot), W_{2}(\cdot) ; y\right)}, \\
i=1,2 .
\end{array}
$$

We denote the $j$ th term of the right-hand side of this equality by $I_{j}, j=1, \ldots, 4$.

Substituting (58) in (46), we see that

$$
\mathbf{H}_{-} \leq H\left(\lambda_{i}(\cdot), W_{j}(\cdot) ; y\right) \leq \mathbf{H}_{+}, \quad i, j=1,2,
$$

where

$$
\mathbf{H}_{-}:=\alpha_{-}{ }^{\sigma-1} \exp (-(\sigma-1) \mathbf{C}), \quad \mathbf{H}_{+}:=\alpha_{+}{ }^{\sigma-1} .
$$

It follows from (5) and Assumption 1 that

$$
\begin{array}{r}
\exp (-(\sigma-1) \mathbf{C}) \leq \exp (-(\sigma-1) c(x, y)) \leq 1 \\
\text { for each } x, y \in D .
\end{array}
$$

Applying this inequality, (7), (45), (56), and (64) to $I_{1}$, we see that

$$
\left\|I_{1}\right\| \leq \|\left|\lambda_{1}(\cdot)-\lambda_{2}(\cdot)\right||| \theta_{1}(\mu, \sigma, \mathbf{C}),
$$

where

$$
\theta_{1}(\mu, \sigma, \mathbf{C}):=\mu\left(\frac{1}{\alpha_{-}}\right)\left(\frac{1}{\mathbf{H}_{-}}\right)
$$

Applying (7), (64), and (66) to $I_{2}$, we see that

$$
\left\|I_{2}\right\| \leq\left\|\lambda_{2}(\cdot)\right\| \mid \theta_{2.1}(\mu, \sigma, \mathbf{C})\left\|W_{1}(\cdot)^{1 / \sigma}-W_{2}(\cdot)^{1 / \sigma}\right\|,
$$

where

$$
\theta_{2.1}(\mu, \sigma, \mathbf{C}):=\mu\left(\frac{1}{\mathbf{H}_{-}}\right)
$$


Making use of (5), (45), (56), and Lemma 7 with $\beta=1 / \sigma$ and $r=1 / \alpha_{+}{ }^{\sigma}$, we obtain the following inequality:

$$
\left\|W_{1}(\cdot)^{1 / \sigma}-W_{2}(\cdot)^{1 / \sigma}\right\| \leq\left\|W_{1}(\cdot)-W_{2}(\cdot)\right\| \theta_{2.2}(\mu, \sigma, \mathbf{C}),
$$

where

$$
\theta_{2.2}(\mu, \sigma, \mathbf{C}):=\left(\frac{1}{\sigma}\right)\left(\frac{1}{\alpha_{+}{ }^{\sigma}}\right)^{1 / \sigma-1} .
$$

Applying this inequality and (43) to (69), we see that

$$
\left\|I_{2}\right\| \leq\left\|W_{1}(\cdot)-W_{2}(\cdot)\right\| \theta_{2}(\mu, \sigma, \mathbf{C}),
$$

where

$$
\theta_{2}(\mu, \sigma, \mathbf{C}):=\theta_{2.1}(\mu, \sigma, \mathbf{C}) \theta_{2.2}(\mu, \sigma, \mathbf{C}) .
$$

Applying (5), (43), (45), (56), (66), and Lemma 7 with $\beta=$ $-(1-1 / \sigma)$ and $r=1 / \alpha_{+}{ }^{\sigma}$ to (57) and performing the same calculations as done in proving (73), we see that

$$
\begin{gathered}
\left\|H\left(\lambda_{1}(\cdot), W_{1}(\cdot) ; \cdot\right)-H\left(\lambda_{1}(\cdot), W_{2}(\cdot) ; \cdot\right)\right\| \\
\quad \leq\left\|W_{1}(\cdot)-W_{2}(\cdot)\right\| \theta_{3.1}(\mu, \sigma, \mathbf{C}),
\end{gathered}
$$

where

$$
\theta_{3.1}(\mu, \sigma, \mathbf{C}):=\left(1-\frac{1}{\sigma}\right)\left(\frac{1}{\alpha_{+} \sigma}\right)^{-(2-1 / \sigma)} .
$$

Combining this inequality and (64), we see that

$$
\left\|\Delta_{1}(\cdot)\right\| \leq\left\|W_{1}(\cdot)-W_{2}(\cdot)\right\| \theta_{3.2}(\mu, \sigma, \mathbf{C}),
$$

where

$$
\theta_{3.2}(\mu, \sigma, \mathbf{C}):=\frac{\theta_{3.1}(\mu, \sigma, \mathbf{C})}{\mathbf{H}_{-}{ }^{2}} .
$$

Applying (7), (42), (45), and (56) to (6), we see that

$$
\begin{aligned}
& \frac{\mu \lambda_{i}(y)}{\alpha_{+}}+(1-\mu) \phi(y) \\
& \leq Y_{\mu}\left(\lambda_{i}(y), W_{j}(y)^{1 / \sigma}\right) \\
& \leq \frac{\mu \lambda_{i}(y)}{\alpha_{-}}+(1-\mu) \phi(y), \quad i, j=1,2 .
\end{aligned}
$$

Integrating both sides of these inequalities with respect to $y \in$ $D$ and making use of (7), (8), (42), and (43), we see that

$$
\begin{array}{r}
\frac{\mu}{\alpha_{+}}+(1-\mu) \leq\left\|\mid Y_{\mu}\left(\lambda_{i}(\cdot), W_{j}(\cdot)^{1 / \sigma}\right)\right\| \leq \frac{\mu}{\alpha_{-}}+(1-\mu), \\
i, j=1,2 .
\end{array}
$$

Applying (7) and (37) to this inequality, we see that

$$
\left\|Y_{\mu}\left(\lambda_{i}(\cdot), W_{j}(\cdot)^{1 / \sigma}\right)\right\| \leq \theta_{3.3}(\mu, \sigma, \mathbf{C}), \quad i, j=1,2,
$$

where

$$
\theta_{3.3}(\mu, \sigma, \mathbf{C}):=\frac{1}{\alpha_{-}}
$$

Applying (66), (77), and (81) to $I_{3}$, we obtain the following inequality:

$$
\left\|I_{3}\right\| \leq\left\|W_{1}(\cdot)-W_{2}(\cdot)\right\| \theta_{3}(\mu, \sigma, \mathbf{C}),
$$

where

$$
\theta_{3}(\mu, \sigma, \mathbf{C}):=\theta_{3.2}(\mu, \sigma, \mathbf{C}) \theta_{3.3}(\mu, \sigma, \mathbf{C})
$$

Performing calculations similar to, but easier than, those done in proving (75), we see that

$$
\begin{aligned}
& \left\|H\left(\lambda_{1}(\cdot), W_{2}(\cdot) ; \cdot\right)-H\left(\lambda_{2}(\cdot), W_{2}(\cdot) ; \cdot\right)\right\| \\
& \quad \leq\|\| \lambda_{1}(\cdot)-\lambda_{2}(\cdot)\|\| \theta_{4.1}(\mu, \sigma, \mathbf{C}),
\end{aligned}
$$

where

$$
\theta_{4.1}(\mu, \sigma, \mathbf{C}):=\left(\alpha_{+}{ }^{\sigma}\right)^{1-1 / \sigma}
$$

We obtain the following inequality by combining this inequality and (64) in the same way as (77):

$$
\left\|\Delta_{2}(\cdot)\right\| \leq\left\|\left|\lambda_{1}(\cdot)-\lambda_{2}(\cdot)\right|\right\| \theta_{4.2}(\mu, \sigma, \mathbf{C}),
$$

where

$$
\theta_{4.2}(\mu, \sigma, \mathbf{C}):=\frac{\theta_{4.1}(\mu, \sigma, \mathbf{C})}{\mathbf{H}_{-}{ }^{2}}
$$

Applying this inequality, (66), and (81) to $I_{4}$, we obtain the following inequality:

$$
\left\|I_{4}\right\| \leq\left\|\left|\lambda_{1}(\cdot)-\lambda_{2}(\cdot) \|\right| \theta_{4}(\mu, \sigma, \mathbf{C}),\right.
$$

where

$$
\theta_{4}(\mu, \sigma, \mathbf{C}):=\theta_{3.3}(\mu, \sigma, \mathbf{C}) \theta_{4.2}(\mu, \sigma, \mathbf{C})
$$

Applying this inequality, (67), (73), and (83) to (62), we obtain the following inequality:

$$
\begin{aligned}
\left\|W_{1}(\cdot)-W_{2}(\cdot)\right\| \leq & \left\|\mid \lambda_{1}(\cdot)-\lambda_{2}(\cdot)\right\| \| \Theta_{1}(\mu, \sigma, \mathbf{C}) \\
& +\left\|W_{1}(\cdot)-W_{2}(\cdot)\right\| \Theta_{2}(\mu, \sigma, \mathbf{C}),
\end{aligned}
$$

where

$$
\begin{aligned}
& \Theta_{1}=\Theta_{1}(\mu, \sigma, \mathbf{C}):=\theta_{1}(\mu, \sigma, \mathbf{C})+\theta_{4}(\mu, \sigma, \mathbf{C}), \\
& \Theta_{2}=\Theta_{2}(\mu, \sigma, \mathbf{C}):=\theta_{2}(\mu, \sigma, \mathbf{C})+\theta_{3}(\mu, \sigma, \mathbf{C}) .
\end{aligned}
$$


Substituting (34) in the definitions of $\theta_{i}=\theta_{i}(\mu, \sigma, \mathbf{C}), i=$ $1, \ldots, 4$, we rewrite $\Theta_{i}=\Theta_{i}(\mu, \sigma, \mathbf{C}), i=1,2$, as follows in the same way as $[12,(5.8),(5.9),(7.19),(7.21)-(7.25)]$ :

$$
\begin{aligned}
& \Theta_{1}(\mu, \sigma, \mathbf{C}) \\
&=\left\{\frac{(1-\mu)}{(\exp (-\alpha)-\mu)}\right\}^{\sigma} \\
& \cdot\left\{Q(\exp (\alpha))^{\sigma-1} \exp (2 \alpha)+\mu \exp (\alpha)\right\}, \\
& \Theta_{2}(\mu, \sigma, \mathbf{C}) \\
&=\left(\frac{\mu}{\sigma}\right) Q(\exp (\alpha))^{\sigma-1} \exp (\alpha) \\
&+\left(1-\frac{1}{\sigma}\right) Q(\exp (\alpha))^{2 \sigma-1} \exp (2 \alpha),
\end{aligned}
$$

where

$$
Q=Q(v):=\frac{(v-\mu)}{\left(v^{-1}-\mu\right)}, \quad \alpha:=(\sigma-1) C .
$$

It is proved in $[12$, Lemma 7.2, (7.22)] that (5), (7), and (22) imply the following inequality:

$$
\Theta_{2}(\mu, \sigma, \mathbf{C})<1 .
$$

Making use of (5), (7), and (36), we see easily that

$$
\Theta_{1}(\mu, \sigma, \mathbf{C})>1 \text {. }
$$

Hence, it follows from (91) and (95) that

$$
\left\|W_{1}(\cdot)-W_{2}(\cdot)\right\| \leq\left\|\left|\lambda_{1}(\cdot)-\lambda_{2}(\cdot)\right|\right\| \theta_{5}(\mu, \sigma, \mathbf{C}),
$$

where

$$
\theta_{5}(\mu, \sigma, \mathbf{C}):=\frac{\Theta_{1}(\mu, \sigma, \mathbf{C})}{\left(1-\Theta_{2}(\mu, \sigma, \mathbf{C})\right)}>0 .
$$

Making use of this inequality, (56), and (71) and defining

$$
\gamma_{1}(\mu, \sigma, \mathbf{C}):=\theta_{2.2}(\mu, \sigma, \mathbf{C}) \theta_{5}(\mu, \sigma, \mathbf{C}),
$$

we obtain Lemma 8.

Proposition 9. If $\mu, \sigma$, and $\mathbf{C}$ satisfy (5), (7), and (22), then the nominal wage equation (3) has a unique solution $w=$ $w(x)$ in $L_{+}^{\infty}(D)$.

Proof. Recalling that (36) follows from (5), (7), and (22) and combining Proposition 6 and Lemma 8 with $\lambda_{1}(x)=\lambda_{2}(x)$, we obtain this proposition.

Remark 10. (i) If $D$ is not a continuous domain but a finite set of points, then the nominal wage equation is not a nonlinear integral equation but a nonlinear equation in an Euclidean space whose dimension is equal to the number of points of $D$ (see $[12,(2.5)])$. This subject is treated in [12]. In [12] we prove a result similar to Propositions 6 and 9 by analyzing this nonlinear equation in the Euclidean space. However, in this paper, Propositions 6 and 9 are proved in the Banach spaces in contrast to the finite dimensional proof done in [12]. Propositions 6 and 9 are similar to, but essentially different from, the results obtained in [12].

(ii) The inequalities (5), (7), and (36) are a sufficient condition for the nominal wage equation (3) to have a solution in $L^{\infty}{ }_{+}(D)$ (see Proposition 6). The inequalities (5), (7), and (22) are a sufficient condition for this solution to be unique. We make use of (22) in order to obtain (95) in the proof of Lemma 8.

(iii) It is proved in [14, Theorem 3.1, (iii)] that if $\sigma>1$ and $\mathbf{C}>0$ are sufficiently small, then (3) has a unique solution. However, the condition (22) is not accepted in [14]. Recalling (35), we see that the result of [14] can be regarded as a corollary of Proposition 9.

By Proposition 9 we can define an operator that maps $\lambda=$ $\lambda(x)$ to $w=w(x)$, where $\lambda=\lambda(x)$ satisfies (42) and (43). We denote this operator by

$$
P_{1}=P_{1}(\lambda(\cdot) ; x)
$$

that is, $w(x):=P_{1}(\lambda(\cdot) ; x)$ satisfies (3). Applying this operator to (10), we can define an operator that maps $\lambda=\lambda(x)$ to $\omega=$ $\omega(x)$. We denote this operator by $P_{2}=P_{2}(\lambda(\cdot) ; x)$; that is, we define

$$
P_{2}(\lambda(\cdot) ; x):=\frac{P_{1}(\lambda(\cdot) ; x)}{G_{\sigma}\left(\lambda(\cdot), P_{1}(\lambda(\cdot) ; \cdot) ; x\right)^{\mu}} .
$$

Proposition 11. If $\lambda_{i}=\lambda_{i}(x), i=1,2$, satisfy (42) and (43), then

$$
\begin{aligned}
& \left\|P_{j}\left(\lambda_{1}(\cdot) ; \cdot\right)-P_{j}\left(\lambda_{2}(\cdot) ; \cdot\right)\right\| \\
& \quad \leq\|\| \lambda_{1}(\cdot)-\lambda_{2}(\cdot)\|\| \gamma_{j}(\mu, \sigma, \mathbf{C}), \quad j=1,2,
\end{aligned}
$$

where $\gamma_{2}=\gamma_{2}(\mu, \sigma, \mathbf{C})$ is a positive-valued function of $(\mu, \sigma, \mathbf{C}) \in(0,1) \times(1,+\infty) \times(0,+\infty)$ (see (99) for $\gamma_{1}=$ $\left.\gamma_{1}(\mu, \sigma, \mathbf{C})\right)$.

Proof. For $j=1$ the result follows form Lemma 8. Next, we prove the case $j=2$. Let us define

$$
\begin{array}{ll}
w_{i}(x):=P_{1}\left(\lambda_{i}(\cdot) ; x\right), & i=1,2, \\
\omega_{i}(x):=P_{2}\left(\lambda_{i}(\cdot) ; x\right), & i=1,2 .
\end{array}
$$

We define $W_{i}=W_{i}(x), i=1,2$, by (103) in the same way as (56). Making use of (75), (85), and (97), we see that

$$
\begin{aligned}
& \left\|H\left(\lambda_{1}(\cdot), W_{1}(\cdot) ; \cdot\right)-H\left(\lambda_{2}(\cdot), W_{2}(\cdot) ; \cdot\right)\right\| \\
& \quad \leq\|\| \lambda_{1}(\cdot)-\lambda_{2}(\cdot)\|\| \theta_{6}(\mu, \sigma, \mathbf{C})
\end{aligned}
$$

where

$$
\theta_{6}(\mu, \sigma, \mathbf{C}):=\theta_{4.1}(\mu, \sigma, \mathbf{C})+\theta_{3.1}(\mu, \sigma, \mathbf{C}) \theta_{5}(\mu, \sigma, \mathbf{C})
$$


Substitute (58) and $(\lambda, \omega, w)=\left(\lambda_{i}, \omega_{i}, w_{i}\right), i=1,2$, in (10). Subtracting both sides of the equalities thus obtained from each other, we obtain

$$
\begin{aligned}
& \omega_{1}(x)-\omega_{2}(x) \\
&=\left(w_{1}(x)-w_{2}(x)\right) H\left(\lambda_{1}(\cdot), W_{1}(\cdot) ; x\right)^{\mu /(\sigma-1)} \\
&+w_{2}(x)\left\{H\left(\lambda_{1}(\cdot), W_{1}(\cdot) ; x\right)^{\mu /(\sigma-1)}\right. \\
&\left.-H\left(\lambda_{2}(\cdot), W_{2}(\cdot) ; x\right)^{\mu /(\sigma-1)}\right\} .
\end{aligned}
$$

Apply (5), (7), (42), (43), (45), (64), (105), and Lemmas 8 and 7 with $\beta=\mu /(\sigma-1)$ and $g_{i}(x)=H\left(\lambda_{i}(\cdot), W_{i}(\cdot) ; x\right), i=1,2$, to the right-hand side of this equality. Defining

$$
\begin{aligned}
\gamma_{2}(\mu, \sigma, \mathbf{C}):= & \gamma_{1}(\mu, \sigma, \mathbf{C}) \mathbf{H}_{+}{ }^{\mu /(\sigma-1)} \\
& +\left(\frac{1}{\alpha_{-}}\right) h\left(\frac{\mu}{(\sigma-1)}, \mathbf{H}_{-}, \mathbf{H}_{+}\right) \theta_{6}(\mu, \sigma, \mathbf{C}),
\end{aligned}
$$

we obtain the present lemma when $j=2$.

Remark 12. (i) We can prove Propositions 6, 9, and 11 with no boundary condition on $\lambda=\lambda(x), w=w(x)$, and $\omega=\omega(x)$. We make use of (42) and (43) only. See [14, Remark 2.3, (i)].

(ii) For simplicity we omit the time variable $t$ in this section. By replacing $\lambda=\lambda(x), w=w(x)$, and $\omega=\omega(x)$ by $\lambda=\lambda(t, x), w=w(t, x)$, and $\omega=\omega(t, x)$ respectively, we make use of Propositions 6, 9, and 11 in the next section.

\section{The Iteration Scheme}

The purpose of this section is to prove Theorem 4. Let us construct an iteration scheme to obtain a solution to the initial value problem for the dcDSK system (3), (10), and (11). Let $\Delta t$ be a constant such that (see (33))

$$
0<\Delta t \leq \frac{1}{\mathbf{a}}
$$

Decompose the time interval $[0,+\infty)$ into an infinite number of intervals $\left[t_{n}, t_{n+1}\right), n \in \mathbb{N} \cup\{0\}$, where

$$
t_{n}:=n \Delta t, \quad n \in \mathbb{N} \cup\{0\} .
$$

Let us define $\lambda_{\Delta t}=\lambda_{\Delta t}(t, x)$ by the following iteration scheme (see (15)):

$$
\begin{gathered}
\lambda_{\Delta t}(0, x):=\lambda^{0}(x), \\
\lambda_{\Delta t}(t, x):=\lambda_{\Delta t}\left(t_{n}, x\right)+M_{\Delta t}\left(t_{n}, x\right) \lambda_{\Delta t}\left(t_{n}, x\right)\left(t-t_{n}\right), \\
\text { for } t \in\left[t_{n}, t_{n+1}\right), \quad n \in \mathbb{N} \cup\{0\},
\end{gathered}
$$$$
\lambda_{\Delta t}\left(t_{n+1}, x\right)=\lambda_{\Delta t}\left(t_{n+1}-0, x\right),
$$

for each $n \in \mathbb{N} \cup\{0\}$, where we define (see (12)-(14))

$$
\begin{gathered}
M_{\Delta t}(t, x):=M\left(\lambda_{\Delta t}(t, \cdot), \omega_{\Delta t}(t, \cdot) ; x\right), \quad t \geq 0, \\
w_{\Delta t}(t, x):=P_{1}\left(\lambda_{\Delta t}(t, \cdot) ; x\right), \\
\omega_{\Delta t}(t, x):=P_{2}\left(\lambda_{\Delta t}(t, \cdot) ; x\right), \\
t \geq 0 .
\end{gathered}
$$

Lemma 13. For each $T>0$, the following ( $i-v i i i)$ statements hold.

(i) $\lambda_{\Delta t}=\lambda_{\Delta t}(t, x) \in L_{0+}^{\infty, 1}([0, T] \times D)$.

(ii) ||$\left|\lambda_{\Delta t}(t, \cdot)\right| \|=1$ for each $t \in[0, T]$.

(iii) $\omega_{\Delta t}=\omega_{\Delta t}(t, x), w_{\Delta t}=w_{\Delta t}(t, x) \in C_{+}(D)$ for each $t \in[0, T]$.

(iv) $1 / \alpha_{+} \leq w_{\Delta t}(t, x) \leq 1 / \alpha_{-}$for each $(t, x) \in[0, T] \times D$.

(v) $1 / \alpha_{+} \leq G_{\sigma}\left(\lambda_{\Delta t}(t, \cdot), w_{\Delta t}(t, \cdot) ; x\right) \leq\left(1 / \alpha_{-}\right) \exp (\mathbf{C})$ for each $(t, x) \in[0, T] \times D$.

(vi) $\mathbf{a}_{1} \leq \omega_{\Delta t}(t, x) \leq \mathbf{a}_{2}$ for each $(t, x) \in[0, T] \times D$.

(vii) $\mathbf{a}_{1} \leq m\left(\lambda_{\Delta t}(t, \cdot), \omega_{\Delta t}(t, \cdot)\right) \leq \mathbf{a}_{2}$ for each $t \in[0, T]$.

(viii) $-\mathbf{a} \leq M_{\Delta t}(t, x) \leq \mathbf{a}$ for each $(t, x) \in[0, T] \times D$.

Proof. By (111) and Assumption 2, we can consider that (i) and (ii) hold when $T=0$. Hence, making use of (115) and Propositions 6 and 9, we obtain (iii)-(viii) when $T=0$. Applying (i), (ii), and (viii) with $T=0,(109)$, and (110) to (112) and (113) when $n=0$, we obtain (i) with $T=t_{1}$. Integrating both sides of (112) with respect to $x \in D$, recalling (12) and (13), and making use of (i) with $T=t_{1}$, we obtain (ii) with $T=t_{1}$. Making use of (i) and (ii) with $T=t_{1}$, (115), and Propositions 6 and 9, we obtain (iii)-(viii) when $T=t_{1}$. Assume that (i) - (viii) hold when $T=t_{k}, k \in \mathbb{N}$. We can prove (i)-(viii) with $T=t_{k+1}$ in the same way as in obtaining (i)(viii) with $T=t_{1}$ from (i)-(viii) with $T=0$. Therefore we prove this lemma.

Note that Propositions 6 and 9 are proved on the basis of (42) and (43). Hence, we need Lemma 13, (i), (ii), in order to make use of Propositions 6 and 9 in the proof of Lemma 13, (iii)-(viii).

Differentiating both sides of (112) with respect to $t \in$ $\left(t_{n}, t_{n+1}\right)$, we obtain the following equality:

$$
\left(\frac{\partial}{\partial t}\right) \lambda_{\Delta t}(t, x)=M_{\Delta t}(t, x) \lambda_{\Delta t}(t, x)+R_{\Delta t}(t, x),
$$

for each $t \in\left(t_{n}, t_{n+1}\right), \quad n \in \mathbb{N} \cup\{0\}$,

where

$$
\begin{aligned}
R_{\Delta t}(t, x):= & -M_{\Delta t}(t, x)\left(\lambda_{\Delta t}(t, x)-\lambda_{\Delta t}\left(t_{n}, x\right)\right) \\
& -\left(M_{\Delta t}(t, x)-M_{\Delta t}\left(t_{n}, x\right)\right) \lambda_{\Delta t}\left(t_{n}, x\right) .
\end{aligned}
$$

Lemma 14. $\left\|\mid R_{\Delta t}(t, \cdot)\right\| \| \mathbf{b} \Delta t$ for each $t \in\left[t_{n}, t_{n+1}\right), n \in$ $\mathbb{N} \cup\{0\}$, where

$$
\mathbf{b}:=\mathbf{a}^{2}+\mathbf{a c}, \quad \mathbf{c}:=2 \gamma_{2}(\mu, \sigma, \mathbf{C})+\mathbf{a}_{2} .
$$


Proof. Subtract both sides of (114) with $t=t_{n}$ from those of (114). Applying (12) and (13) to the equality thus obtained, we obtain

$$
\begin{aligned}
& M_{\Delta t}(t, x)-M_{\Delta t}\left(t_{n}, x\right) \\
& =\omega_{\Delta t}(t, x)-\omega_{\Delta t}\left(t_{n}, x\right) \\
& \quad-\left(\int_{y \in D} \lambda_{\Delta t}(t, y) \omega_{\Delta t}(t, y) d y\right. \\
& \left.\quad-\int_{y \in D} \lambda_{\Delta t}\left(t_{n}, y\right) \omega_{\Delta t}\left(t_{n}, y\right) d y\right) .
\end{aligned}
$$

Applying Lemma 13, (ii), (vi), and Proposition 11 with $j=2$ and $\left(\lambda_{1}(x), \lambda_{2}(x)\right)=\left(\lambda_{\Delta t}(t, x), \lambda_{\Delta t}\left(t_{n}, x\right)\right)$ to the right-hand side of this equality, we see that

$$
\begin{array}{r}
\left\|M_{\Delta t}(t, \cdot)-M_{\Delta t}\left(t_{n}, \cdot\right)\right\| \leq \mathbf{c}\left\|\lambda_{\Delta t}(t, \cdot)-\lambda_{\Delta t}\left(t_{n}, \cdot\right)\right\| \| \\
\qquad \text { for each } t \in\left[t_{n}, t_{n+1}\right), \quad n \in \mathbb{N} \cup\{0\} .
\end{array}
$$

Applying Lemma 13, (ii), (viii), and (110) to (112), we see that

$$
\left\|\lambda_{\Delta t}(t, \cdot)-\lambda_{\Delta t}\left(t_{n}, \cdot\right)\right\| \mid \leq \mathbf{a} \Delta t .
$$

Applying this inequality, Lemma 13, (ii), (viii), and (120) to (117), we obtain the present lemma.

Lemma 15. The function

$$
\left(\lambda_{\Delta t}(t, x), \omega_{\Delta t}(t, x), w_{\Delta t}(t, x)\right)
$$

converges in (17) as $\Delta t \rightarrow 0+0$ for each $T>0$.

Proof. Replace $\Delta t$ by $\Delta s$ in (116), where $\Delta s$ is a positive constant that satisfies the same inequality as (109). Subtracting both sides of the equality thus obtained from those of (116), we obtain

$$
\left(\frac{\partial}{\partial t}\right) \Lambda_{\Delta t, \Delta s}(t, x)=M_{\Delta t}(t, x) \Lambda_{\Delta t, \Delta s}(t, x)+\mathbf{R}_{\Delta t, \Delta s}(t, x),
$$

where

$$
\begin{aligned}
\Lambda_{\Delta t, \Delta s}(t, x) & :=\lambda_{\Delta t}(t, x)-\lambda_{\Delta s}(t, x), \\
\mathbf{R}_{\Delta t, \Delta s}(t, x) & :=R_{\Delta t}(t, x)-R_{\Delta s}(t, x)+r_{\Delta t, \Delta s}(t, x), \\
r_{\Delta t, \Delta s}(t, x) & :=\left(M_{\Delta t}(t, x)-M_{\Delta s}(t, x)\right) \lambda_{\Delta s}(t, x) .
\end{aligned}
$$

Performing the same calculations as done in proving (120) and making use of Lemma 13, (ii), we obtain

$$
\left\|\left|r_{\Delta t, \Delta s}(t, \cdot) \|\right| \leq \mathbf{c k}(t)\right.
$$

where

$$
\mathbf{k}(t):=\left\|\Lambda_{\Delta t, \Delta s}(t, \cdot)\right\| \mid .
$$

Applying this inequality and Lemma 14 to (125), we deduce that

$$
\left\|\mathbf{R}_{\Delta t, \Delta s}(t, \cdot)\right\| \mid \leq \mathbf{b}(\Delta s+\Delta t)+\mathbf{c k}(t) .
$$

Let us solve (123) with respect to (124) by considering (125) as a perturbation term. We perform the same calculations as done in proving Gronwall's lemma. Replace $t$ by $r$ in (123), multiply both sides by

$$
\exp \left(-\int_{0}^{r} M_{\Delta t}(s, x) d s\right)
$$

and integrate both sides with respect to $r \in[0, t]$. Applying Lemma 13, (viii), and (129) to the equality thus obtained and noting that $\Lambda_{\Delta t, \Delta s}(0, x)=0$ (see (111)), we can easily obtain an integral inequality for $\mathbf{k}(t)$. Consider this integral inequality as a differential inequality whose unknown function is

$$
\mathbf{K}=\mathbf{K}(t):=\int_{0}^{t} \mathbf{k}(r) e^{-\mathbf{a} r} d r
$$

Solve this differential inequality with respect to $\mathbf{K}=\mathbf{K}(t)$. Applying the inequality thus solved to the integral inequality for $\mathbf{k}=\mathbf{k}(t)$, we obtain

$$
\|\| \lambda_{\Delta t}(t, \cdot)-\lambda_{\Delta s}(t, \cdot) \||| \leq \mathbf{r}(t)(\Delta t+\Delta s)
$$

where

$$
\mathbf{r}(t):=\left(\frac{\mathbf{b}}{(\mathbf{a}+\mathbf{c})}\right)\{\exp ((\mathbf{a}+\mathbf{c}) t)-1\}
$$

Applying this inequality and Proposition 11 with $\left(\lambda_{1}(x), \lambda_{2}(x)\right)=\left(\lambda_{\Delta t}(t, x), \lambda_{\Delta s}(t, x)\right)$ to (115), we deduce that

$$
\begin{gathered}
\left\|w_{\Delta t}(t, \cdot)-w_{\Delta s}(t, \cdot)\right\| \leq \gamma_{1}(\mu, \sigma, \mathbf{C}) \mathbf{r}(t)(\Delta t+\Delta s), \\
\left\|\omega_{\Delta t}(t, \cdot)-\omega_{\Delta s}(t, \cdot)\right\| \leq \gamma_{2}(\mu, \sigma, \mathbf{C}) \mathbf{r}(t)(\Delta t+\Delta s) .
\end{gathered}
$$

From (132)-(134), we obtain the present lemma.

Lemma 16. The limit

$$
\begin{aligned}
& (\lambda(t, x), \omega(t, x), w(t, x)) \\
& \quad:=\lim _{\Delta t \rightarrow 0+0}\left(\lambda_{\Delta t}(t, x), \omega_{\Delta t}(t, x), w_{\Delta t}(t, x)\right)
\end{aligned}
$$

is a global solution and satisfies (24), (25), and (27)-(32).

Proof. Making use of Lemmas 13 and 15, we deduce that (135) satisfies (25) and (28)-(32). Recalling (115), we can substitute (122) in (3) and (10). Let $\Delta t \rightarrow 0+0$ in the equalities thus obtained. By Lemma 15, we see easily that (135) satisfies (3) and (10). Replace $t$ by $r$ in (116). Multiply both sides by (130) and integrate both sides with respect to $r \in[0, t]$. We obtain an integral equation whose unknown function is $\lambda_{\Delta t}=\lambda_{\Delta t}(t, x)$. Apply (111) and Lemmas 14 and 15 to this integral equation, recall (12) and (13), and let $\Delta t \rightarrow 0+0$. We deduce that (135) satisfies the following equality:

$$
\lambda(t, x)=\lambda^{0}(x) \exp \left(\int_{0}^{t} M(\lambda(s, \cdot), \omega(s, \cdot) ; x) d s\right) .
$$

Hence, (135) satisfies (11) and (15) (see (14)). Applying (32) to (136), we see that (135) satisfies (24) and (27). 
The following lemma gives necessary conditions for the initial value problem to have a solution that belongs to (17).

Lemma 17. If the initial value problem has a solution

$$
(\lambda, \omega, w)=(\lambda(t, x), \omega(t, x), w(t, x))
$$

in (17) for some $T>0$, then this solution satisfies (23), (25), (26), and (28)-(32) in $[0, T] \times D$, where $\delta_{i}(\mu, \sigma, \mathbf{C}):=$ $\mathbf{a} \gamma_{i}(\mu, \sigma, \mathbf{C}), i=1,2$ (see Proposition 11).

Proof. Recalling (12), (13), and the definition of (17), we see easily that

$$
\begin{gathered}
\omega(t, x) \in L^{\infty}([0, T] \times D), \quad m(t) \in L^{\infty}([0, T]), \\
\lambda(t, x) \in L_{0+}^{\infty, 1}([0, T] \times D),
\end{gathered}
$$

where

$$
m(t):=m(\lambda(t, \cdot), \omega(t, \cdot))
$$

Hence, integrating both sides of (11) with respect to $x \in D$ and recalling (12) and (13), we see easily that

$$
\frac{d n(t)}{d t}=m(t)(1-n(t))
$$

where

$$
n(t):=\||\lambda(t, \cdot) \|| \mid
$$

Making use of (138) and (139), we can transform (11) into (136). Applying (138) and Assumption 2, (ii), to (136), we obtain

$$
n(0+0)=1
$$

Solving (141) with this initial condition, we see that $\lambda=\lambda(t, x)$ satisfies (28) for each $t \in[0, T]$. Making use of this result and (139), we can apply Propositions 6, 9, and 11 to (137). Applying Propositions 6 and 9 to (137), we see that (137) satisfies (25) and (29)-(32) for each $(t, x) \in[0, T] \times D$. Integrate both sides of (11) with respect to $t \in[r, s]$, calculate the absolute values of both sides, and integrate them with respect to $x \in D$. Applying (28) and (32) to the right-hand side of the equality thus obtained, we see that (137) satisfies (23) for each $r, s \in$ $[0, T]$. Combining this result and Proposition 11, we see that (137) satisfies (26) for each $r, s \in[0, T]$.

Proof of Theorem 4. Let

$$
\left(\lambda_{1}, \omega_{1}, w_{1}\right)=\left(\lambda_{1}(t, x), \omega_{1}(t, x), w_{1}(t, x)\right)
$$

be a solution that belongs to (17). Substitute this solution and (135) in (11). Subtracting both sides of the equalities thus obtained from each other, we obtain the following equation in the same way as (123):

$$
\left(\frac{\partial}{\partial t}\right) \Lambda(t, x)=M(t, x) \Lambda(t, x)+\mathbf{r}(t, x),
$$

where

$$
\begin{gathered}
\Lambda(t, x):=\lambda(t, x)-\lambda_{1}(t, x), \\
\mathbf{r}(t, x):=\left(M(t, x)-M_{1}(t, x)\right) \lambda_{1}(t, x), \\
M(t, x):=M(\lambda(t, \cdot), \omega(t, \cdot) ; x), \\
M_{1}(t, x):=M\left(\lambda_{1}(t, \cdot), \omega_{1}(t, \cdot) ; x\right) .
\end{gathered}
$$

Note that both (135) and (144) satisfy (25) and (28)-(32) (see Lemmas 16 and 17). Making use of this result, we can perform the same calculations as done in proving (127). Hence we obtain

$$
\|\mathbf{r}(t, \cdot)\||\leq \mathbf{c}\||\Lambda(t, \cdot)|\| .
$$

Making use of this inequality in place of (129) and performing the same calculations as done in proving (132)-(134), we prove that (144) is the same as (135). By Lemmas 16 and 17 we prove Theorem 4 .

\section{Conflict of Interests}

The authors declare that there is no conflict of interests regarding the publication of this paper.

\section{Acknowledgments}

This work was supported by Grant-in-aid for Scientific Research (22530242), Ministry of Education, Culture, Sports, Science and Technology of Japan. The authors would like to express their deepest gratitude to the anonymous reviewer for his/her valuable suggestions.

\section{References}

[1] M. Fujita, P. Krugman, and A. J. Venables, The Spatial Economy, The MIT Press, Boston, Mass, USA, 1999.

[2] M. Fujita and J.-F. Thisse, Economics of Agglomeration, Cities, Industrial Location, and Regional Growth, Cambridge University Press, 2002.

[3] M. Fujita, "The evolution of spatial economics: from Thünen to the new economic geography," The Japanese Economic Review, vol. 61, no. 1, pp. 1-32, 2010.

[4] P. Krugman, "Increasing returns and economic geography," The Journal of Political Economy, vol. 99, no. 3, pp. 483-499, 1991.

[5] P. Krugman, The Self-Organizing Economy, Blackwell, London, UK, 1996.

[6] P. Krugman, "The official web site of the Nobel Prize in Economic Sciences," http://www.nobelprize.org/nobel_prizes/ economics/laureates/2008/.

[7] S. B. S. D. Castro, J. Correia-da-Silva, and P. Mossay, "The core-periphery model with three regions and more," Papers in Regional Science, vol. 91, no. 2, pp. 401-418, 2012.

[8] P. Mossay, "Increasing returns and heterogeneity in a spatial economy," Regional Science and Urban Economics, vol. 33, no. 4, pp. 419-444, 2003.

[9] P. Mossay, "The core-periphery model: a note on the existence and uniqueness of short-run equilibrium," Journal of Urban Economics, vol. 59, no. 3, pp. 389-393, 2006. 
[10] P. Mossay and T. Tabuchi, "Preferential trade agreements harm third countries," CIRJE Discussion Paper No. F-811, University of Tokyo, 2011.

[11] N. G. Pavlidis, M. N. Vrahatis, and P. Mossay, "Existence and computation of short-run equilibria in economic geography," Applied Mathematics and Computation, vol. 184, no. 1, pp. 93103, 2007.

[12] M. Tabata, N. Eshima, Y. Kiyonari, and I. Takagi, “The existence and uniqueness of short-run equilibrium of the Dixit-StiglitzKrugman model in an urban-rural setting," IMA Journal of Applied Mathematics, 2015.

[13] M. Tabata, N. Eshima, and Y. Sakai, "Existence, uniqueness, and computation of short-run and long-run equilibria of the Dixit-Stiglitz-Krugman model in an urban setting," Applied Mathematics and Computation, vol. 234, pp. 339-355, 2014.

[14] M. Tabata, N. Eshima, Y. Sakai, and I. Takagi, "An extension of Krugman's core-periphery model to the case of a continuous domain: existence and uniqueness of solutions of a system of nonlinear integral equations in spatial economics," Nonlinear Analysis B: Real World Applications, vol. 14, no. 6, pp. 2116-2132, 2013.

[15] J. Hofbauer and K. Sigmund, The Theory of Evolution and Dynamical Systems: Mathematical Aspects of Selection, Cambridge University Press, 1988.

[16] J. W. Weibull, Evolutionary Game Theory, The MIT Press, Cambridge, Mass, USA, 1995.

[17] P. A. Samuelson, "The transfer problem and transport costs: the terms of trade when impediments are absent," The Economic Journal, vol. 62, pp. 278-304, 1952. 


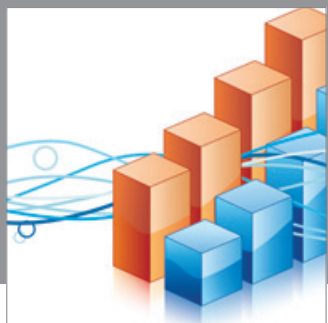

Advances in

Operations Research

mansans

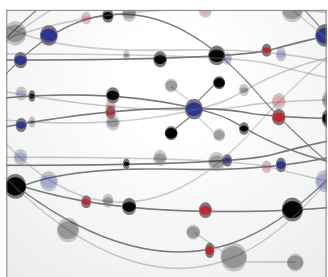

The Scientific World Journal
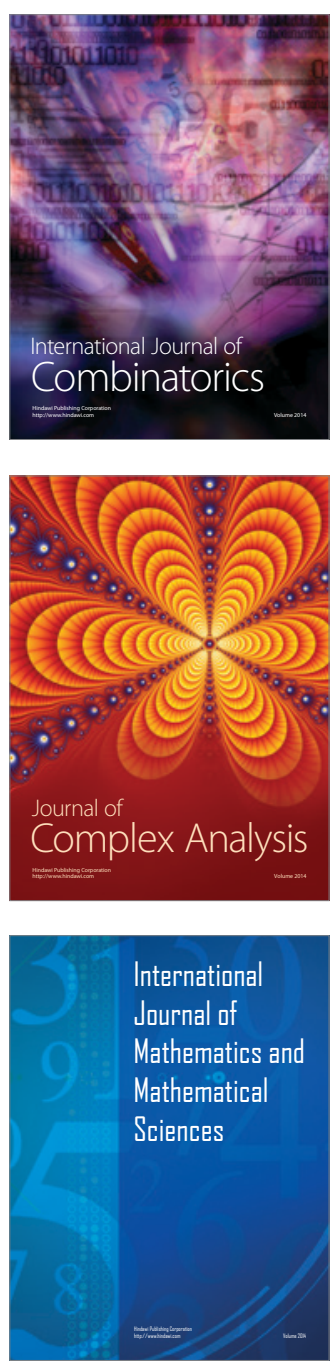
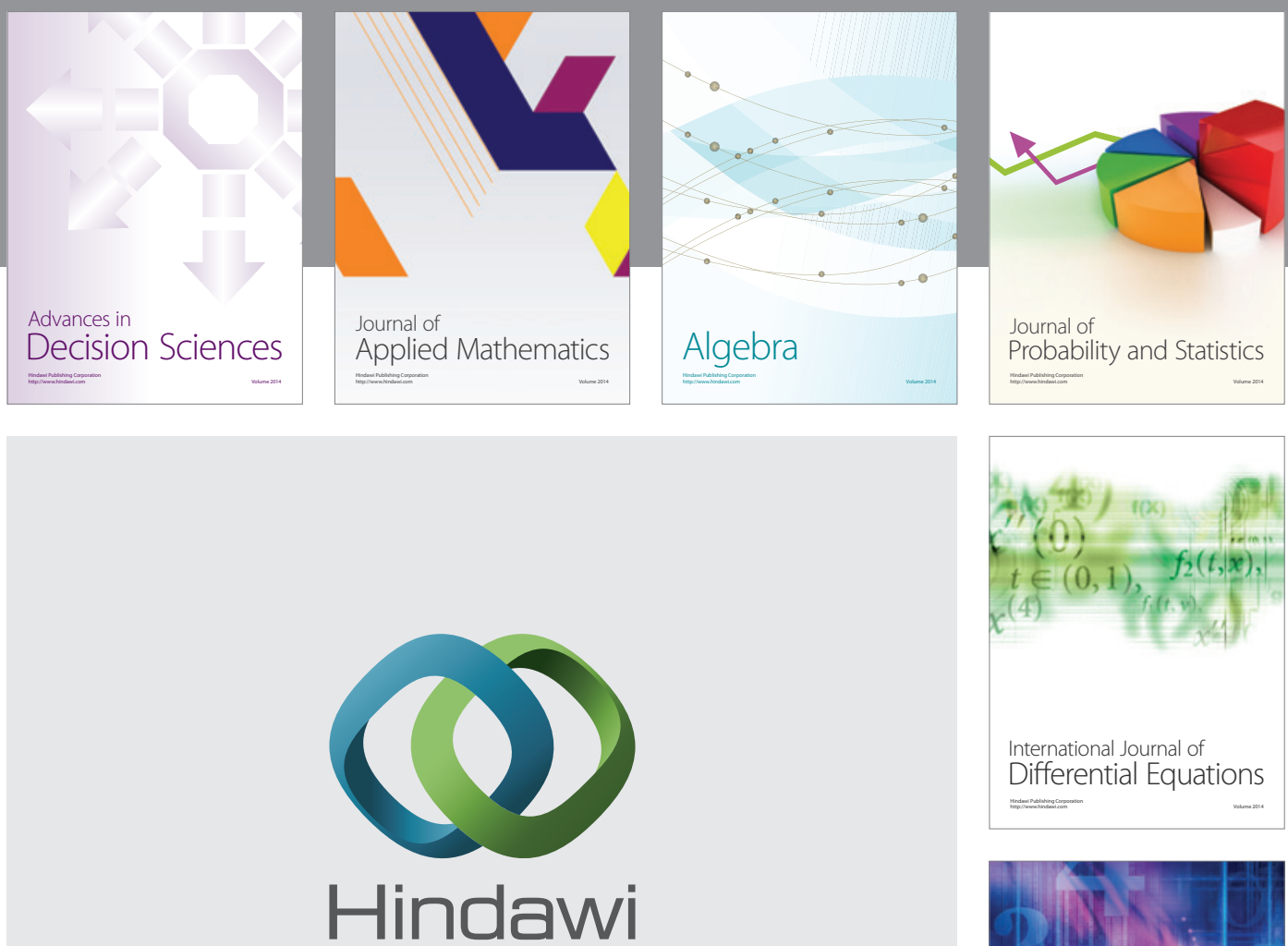

Submit your manuscripts at http://www.hindawi.com
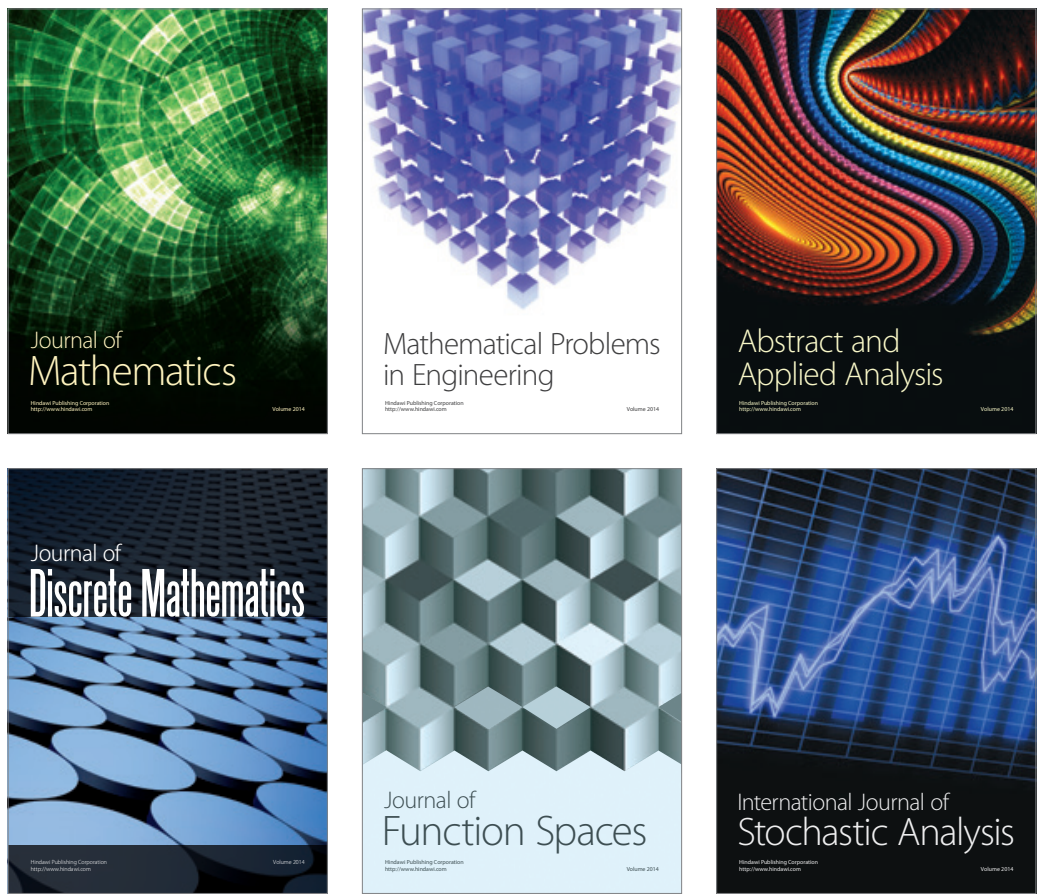

Journal of

Function Spaces

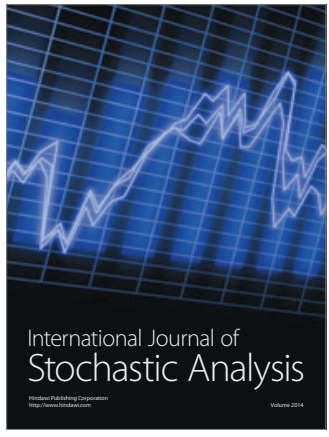

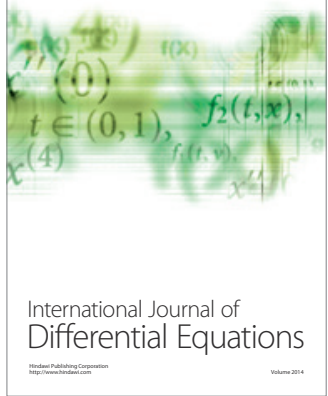
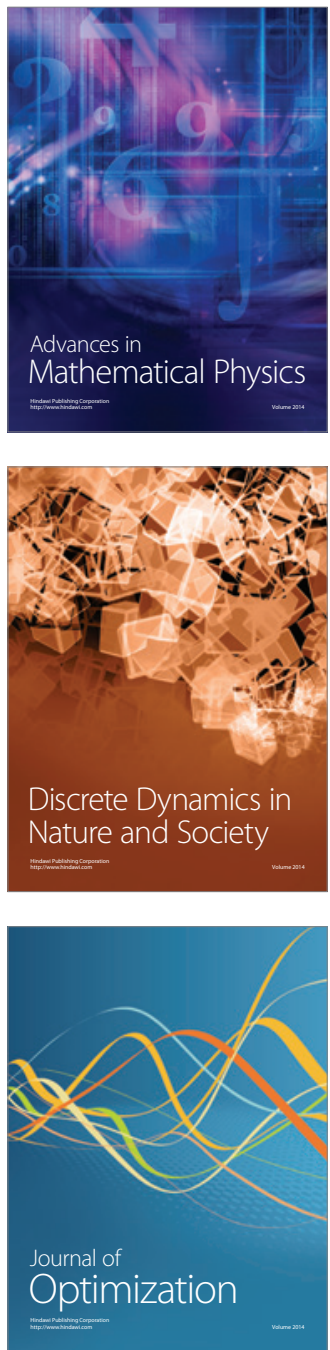